\title{
Symptoms and Transmission of COVID-19 Infection in Children
}

\author{
Celal Özcan ${ }^{1}$ and İzzettin Toktaş ${ }^{1}$ \\ ${ }^{1}$ Diyarbakır Children's Hospital
}

October 8, 2020

\begin{abstract}
Objective: Children with COVID-19 are reported to get the infection from an adult contact, with minimal secondary transmission from children. Children do not appear to be efficient transmitters of infection. Common symptoms in adults infected with COVID-19 include fever, dry cough and fatigue. The symptoms in children are more atypical than in adults, and coughing is lighter. Methods: Children aged 0-18 who was admitted to the emergency department from March 20 to September 15, 2020 and had confirmed diagnosis of COVID-19 with a positive real-time reverse-transcriptase polymerase chain reaction (RT-PCR) were included in the study. Results: RT-PCR tests were done in 835 children with suspected SARS-CoV-2 infection and the test was positive in $178(21.3 \%)$ children. The most common symptoms at the onset of illness were fever $(67.4 \%)$, headache $(41.1 \%)$, cough $(36.6 \%)$, sore throat $(25.7 \%)$, fatigue $(22.9 \%)$, myalgia $(22.9 \%)$ and diarrhea $(20.6 \%)$. One hundred and seven $(61.1 \%)$ of the patients were infected by close contact with family members diagnosed with COVID-19, 35 (20\%) of the patients were infected via close contact with non-family members diagnosed with COVID-19, 11 (6.3\%) of the patients had a history of exposure in an epidemic area, including wedding hall, condolence house and shopping center and 23 (13.1\%) of the patients with unknown source of infection. Conclusion: COVID-19 infection is seen to be milder and cause less hospitalizations and patient deaths in children. Crowded indoor environments are the main centers of the spread of the outbreak and children are unlikely to be the main source of infection of the pandemic.
\end{abstract}

\section{Tables: 1}

Key words: COVID-19 infection, children, symptoms, transmission, source of infection, real-time PCR

Conflict of interest : The authors have no financial relationships or other conflict of interests to disclose.

Correspondence : Celal Özcan, MD, Associate Professor of Pediatric Allergy and immunology

Address : Diyarbakır Children's Hospital, Division

Department of Pediatric Allergy and immunology

Yenişehir / Diyarbakır 21100 TURKEY

Tel : +90 5052520448

Fax : +90412 2290147

e-mail : celalozcan01@yahoo.com.tr

\section{List of Authors}

Celal Özcan, Celal Özcan, MD, Associate Professor of Pediatric Allergy and immunology Diyarbakır Children's Hospital, Department of Pediatric Allergy and immunology

Contribution of Author : Conception and design of the study, data generation, preparation and critical revision of the manuscript 
e-mail: celalozcan01@yahoo.com.tr

İzzettin Toktaş, MD, Specialist in Public Health

Diyarbakır Children's Hospital Chief Physician, Diyarbakır, Turkey

Contribution of Author : analysis and interpretation of the data, preparation and critical revision of the manuscript

e-mail: drizzettin@gmail.com

\section{Abstract}

Objective: Children with COVID-19 are reported to get the infection from an adult contact, with minimal secondary transmission from children. Children do not appear to be efficient transmitters of infection. Common symptoms in adults infected with COVID-19 include fever, dry cough and fatigue. The symptoms in children are more atypical than in adults, and coughing is lighter.

Methods: Children aged 0-18 who was admitted to the emergency department from March 20 to September 15, 2020 and had confirmed diagnosis of COVID-19 with a positive real-time reverse-transcriptase polymerase chain reaction (RT-PCR) were included in the study.

Results: RT-PCR tests were done in 835 children with suspected SARS-CoV-2 infection and the test was positive in $178(21.3 \%)$ children. The most common symptoms at the onset of illness were fever $(67.4 \%)$, headache $(41.1 \%)$, cough $(36.6 \%)$, sore throat $(25.7 \%)$, fatigue $(22.9 \%)$, myalgia $(22.9 \%)$ and diarrhea $(20.6 \%)$. One hundred and seven $(61.1 \%)$ of the patients were infected by close contact with family members diagnosed with COVID-19, 35 (20\%) of the patients were infected via close contact with non-family members diagnosed with COVID-19, 11 (6.3\%) of the patients had a history of exposure in an epidemic area, including wedding hall, condolence house and shopping center and $23(13.1 \%)$ of the patients with unknown source of infection.

Conclusion: COVID-19 infection is seen to be milder and cause less hospitalizations and patient deaths in children. Crowded indoor environments are the main centers of the spread of the outbreak and children are unlikely to be the main source of infection of the pandemic.

Key words: COVID-19 infection, children, symptoms, transmission, source of infection, real-time PCR

\section{INTRODUCTION}

Coronavirus Disease 2019 (COVID-19) is a viral disease caused by severe acute respiratory syndrome coronavirus 2 (SARS-CoV-2). Coronaviruses are a large family of viruses that mostly present in mammals and birds (1). Early cases of COVID- humans have a history of going to the Huanan Seafood Wholesale Market in . Therefore, it was initially assumed that animal-to-human transmission was the main mechanism. In a short period of time, cases with no history of visiting the market were reported. Therefore, it was understood that the virus can also be transmitted from human-to-human (2).

The transmission route of COVID-19 disease develops as a result of inhalation of the infected droplet or contact with the surfaces and fomites soiled by infected respiratory secretion during coughing or sneezing of the infected person (3). Comprehensive population-based epidemiological studies have confirmed that SARS-CoV-2 infection is less common in children than in adults and if children do acquire SARS-CoV-2, the vast majority experience mild disease not requiring hospitalization. Children with COVID-19 are reported to get the infection from an adult contact, with minimal secondary transmission from children. Children do not appear to be efficient transmitters of infection (4).

The disease initially presents as a mild respiratory disease and develops into viral interstitial pneumonia, which may progress to acute respiratory distress syndrome (ARDS) and multi-organ failure (MOF) in 10$15 \%$ of adults. The course of the disease is generally mild in children compared to adults, the reason for this has not been clear yet (5). Common symptoms in adults infected with COVID-19 include fever, dry cough 
and fatigue. The symptoms in children are more atypical than in adults, and coughing is lighter. Instead of common symptoms in adults, gastrointestinal symptoms such as diarrhea and vomiting are common in children (1). In this study, we aimed to assess the common symptoms and possible source of infection in pediatric cases with SARS-CoV-2 infection.

\section{METHODS:}

This single-center study was undertaken in Diyarbakır Children's Hospital, the largest children's hospital in . Children aged 0-18 who was admitted to the emergency department from March 20 to September 15, 2020 and had confirmed diagnosis of COVID-19 with a positive real-time reverse-transcriptase polymerase chain reaction (RT-PCR) were included in the study. The families of the patients were called to obtain information about the demographic characteristics, symptoms, comorbid diseases and transmission of COVID-19 Infection. The patients' laboratory results and chest x-ray were retrospectively analyzed in the hospital's electronic database. This study was approved by the ethics committee of the Diyarbakır Gazi Yaşargil Training and .

In order to detect the SARS-CoV-2, nasopharyngeal and nasal swabs were obtained from patients who suspected for COVID-19 disease. These specimens were put immediately in viral transport medium and kept at $4^{*} \mathrm{C}$ during transport. Samples were sent to the Laboratory of Diyarbakır Gazi Yaşargil Training and to be investigated for the presence of SARS-CoV-2 pathogen using a real-time PCR method. Viral nucleic acid extract was obtained from the samples using a kit ().

By calling the families of the children we questioned;

-The symptoms of the child's admission to the hospital,

-Whether children have symptoms during the interview,

-Whether the child was hospitalized or required intensive care unit,

-Who is the first COVID-19 patient in the family,

-From whom may the child have received the COVID-19 infection,

-How many people in the family had positive COVID-19 PCR test,

-Whether there is a family member hospitalized or required intensive care unit due to COVID-19 disease,

- Whether the child has a comorbid disease.

Phone calls were made by one and the same physician. Phone calls were made at the earliest two weeks after the patient's COVID-19 PCR test was positive.

Cough, nasal congestion, sneezing, rhinorrhea, sore throat, and dyspnea were classified as respiratory symptoms, diarrhea, vomiting, nausea and abdominal pain were classified as gastrointestinal symptoms

Statistical analysis was performed using the SPSS packet program. Values were either provided as numbers and percentages, or as mean \pm standard deviation, where applicable. Comparisons of the mean age of patients and mean number of symptoms between patients whose source of infection was outside of family members and whose source of infection was family members were made using the Chi-square test, Fisher's exact test and student's t-test. A p-value of [?] 0.05 was considered indicative of statistical significance.

\section{RESULTS}

\section{Demographic information}

From March 20 to September 15, 2020, RT-PCR tests were done in 835 children with suspected SARS-CoV2 infection and the test was positive in $178(21.3 \%)$ children. Information of three patients could not be obtained. One hundred seventy-five children aged from 4 months to 18 years with mean age of $10.6 \pm 5.4$ years were included in the study. The male/female ratio was 1.01 (88/87). The first COVID-19 patient was 
identified in our hospital on April 7, 2020. A total of 28 COVID-19 patients were identified in approximately 3 months from the beginning to June 30 , while 150 COVID-19 patients were identified in the next 2.5 months.

\section{Symptoms information}

One hundred sixty four $(93.7 \%)$ children were symptomatic whereas $11(6.3 \%)$ children were asymptomatic. The most common symptoms at the onset of illness were fever $(67.4 \%)$, headache $(41.1 \%)$, cough $(36.6 \%)$, sore throat $(25.7 \%)$, fatigue $(22.9 \%)$, myalgia $(22.9 \%)$ and diarrhea $(20.6 \%)$; less common symptoms were vomiting (16\%), dyspnea (14.9\%), nasal congestion (13.1\%), nausea (13.1\%), anosmia $(13.1 \%)$, abdominal pain $(11.4 \%)$, loss of appetite $(11.4 \%)$,rhinorrhea $(9.7 \%)$, loss of taste $(9.1 \%)$, joint pain $(8.6 \%)$, dizziness (8\%), chest pain (4.6\%), sneezing (4\%) and shaking (3.4\%) (Table1). In the interviews made at the first two weeks after the onset of symptoms, no patient's symptoms continued.

Among the symptomatic patients, 24 (14.6\%) patients had only one symptom, 33 (20.1\%) patients had two symptoms, $29(17.7 \%)$ patients had three symptoms, $26(15.9 \%)$ patients had four symptoms, $13(7.9 \%)$ patients had five symptoms, $15(9.1 \%)$ patients had six symptoms, $8(4.9 \%)$ patients had seven symptoms, $3(1.8 \%)$ patients had eight symptoms, $4(2.4 \%)$ patients had nine symptoms, $1(0.6 \%)$ patient had ten symptoms, $2(1.2 \%)$ patients had eleven symptoms, $3(1.8 \%))$ patients had twelve symptoms, $2(1.2 \%)$ patients had thirteen symptoms, $1(0.6 \%)$ patient had fifteen symptoms. The mean number of symptoms was $4.0 \pm 2.8$.

One hundred and two (58.3\%) of the patients had respiratory symptoms, 69 (39.4\%) of the patients had gastrointestinal symptoms, $59(33.7 \%)$ of the patients had only respiratory symptoms, $26(14.9 \%)$ of the patients had only gastrointestinal symptoms and 24 (13.7\%) of the patients had only fever.

Six patients had atypical symptoms. Patient 1; 17-year-old male patient with paralysis of the left leg lasting for 12 days, patient 2; 15-year-old male patient with hoarseness lasting for two weeks, patient 3 and 4; 16-yearold and 12-year-old female patients with backache, patient $5 ; 12$ year old male patient with conjunctivitis, patient 6 ; 17-year-old male patient with painful swelling in the eyes lasting within one week.

\section{Comorbid diseases and outcomes}

Fifteen $(8.6 \%)$ of the patients were hospitalized, three $(1.7 \%)$ of them were required intensive care unit. The hospitalized mean number of days was $6.5 \pm 3.0$ and the required intensive care unit mean number of days

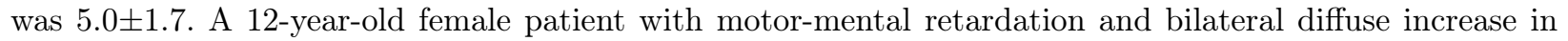
density on chest X-ray was died from SARS-CoV-2 infection.

Twenty eight (16\%) of the patients had comorbid diseases. Twenty (11.4\%) of them had asthma, $4(2.3 \%)$ of them had motor-mental retardation and $4(2.3 \%)$ of them had congenital heart disease. Only two of the patients with asthma were hospitalized and didn't require intensive care unit. Three of the patients with motor-mental retardation were hospitalized and two of them were required intensive care unit. Two of the patients with congenital heart disease were hospitalized and one of them was required intensive care unit.

\section{Epidemiological information}

COVID-19 PCR test was positive in at least one of the family members in 140 (80\%) patients. Up to seven people in a family have tested positive for COVID-19 PCR. The mean number of people who tested positive for COVID-19 PCR was $2.3 \pm 1.4$. At least one family member was hospitalized in 32 (18.3\%) patients, 12 $(6.9 \%)$ of them their mothers, $11(6.3 \%)$ of them their fathers, $6(3.4 \%)$ of them their grandparents and 3 (1.7\%) of them their siblings.

One hundred and seven $(61.1 \%)$ of the patients were infected by close contact with family members diagnosed with COVID-19, 35 (20\%) of the patients were infected via close contact with non-family members diagnosed with COVID-19, $11(6.3 \%)$ of the patients had a history of exposure in an epidemic area, including wedding hall, condolence house and shopping center and 23 (13.1\%) of the patients with unknown source of infection (Table1). Of the family members that are the source of infection, $44(41.1 \%)$ were mothers, $37(34.6 \%)$ were fathers, $14(13.1 \%)$ were grandparents, $12(11.2 \%)$ were siblings 
In half of the 68 patients whose source of infection was outside of family members, nobody had COVID-19 at home after them, but at least one person at home was infected with COVID-19 in the other half.

The mean age of patients whose source of infection was outside of family members and whose source of infection was family members was $12.7 \pm 4.9$ and $9.3 \pm 5.3$ respectively $(\mathrm{p}<0.001)$. At least one family member was hospitalized in 31 patients whose source of infection was family members and at least one family member was hospitalized in patients whose source of infection was outside of family members $(p<0.001)$. The mean number of symptoms of patients whose source of infection was outside of family members and whose source of infection was family members was $4.5 \pm 3.4$ and $3.3 \pm 2.4$ respectively $(\mathrm{p}<0.014)$.

\section{Laboratory information}

Within 175 patients, $61(34.8 \%)$ underwent laboratory testing. Among these patients, leucopenia (White Blood Count $<4.5 \times 10^{9} / \mathrm{L}$ ) in 11 (\%18) patients, lymphocytopenia (lymphocyte count $<1.2 \times 10^{9} / \mathrm{L}$ ) in 12 (\%19.7) patients, neutropenia (neutrophil count $<1.5 \times 10^{9} / \mathrm{L}$ ) in three (4.9\%) patients and thrombocytopenia (platelet count $<150 \times 10^{9} / \mathrm{L}$ ) in five $(8.2 \%)$ patients were detected. Furthermore increases in alanine aminotransferase $(>45 \mathrm{U} / \mathrm{L}$ ) in five patients (minimum $46 \mathrm{U} / \mathrm{L}$, maximum $69 \mathrm{U} / \mathrm{L}$ ) and increases in aspartate aminotransferase $(>50 \mathrm{U} / \mathrm{L}$ ) in three patients (minimum $94 \mathrm{U} / \mathrm{L}$, maximum $115 \mathrm{U} / \mathrm{L}$ ) were detected, while decreases in calcium $(<8.5 \mathrm{mg} / \mathrm{dL}$ ) in nine patients (minimum $6.7 \mathrm{mg} / \mathrm{dL}$, maximum $8.4 \mathrm{mg} / \mathrm{dL}$ ), decreases in sodium $(<135 \mathrm{mEq} / \mathrm{L}$ ) in $13(21.3 \%)$ patients (minimum $129 \mathrm{mEq} / \mathrm{L}$, maximum $134 \mathrm{mEq} / \mathrm{L}$ ) and decreases in potassium $(<3.5 \mathrm{mEq} / \mathrm{L}$ ) in three patients (minimum $3 \mathrm{mEq} / \mathrm{L}$, maximum $3.4 \mathrm{mEq} / \mathrm{L}$ ) were detected.

\section{Imaging information}

Fifty-two patients were underwent chest X-ray. Lung findings were detected in 19 (36.5\%) patients. It was unilateral localized increase in density in seven patients on the right lower zone, bilateral localized increase in density in six patients, unilateral mild bronchial thickening in five patients on the right zone and bilateral diffuse increase in density in one patient.

\section{DISCUSSION}

There are data from Europe, Asia and America confirming that the proportion of children infected with COVID-19 in the community is low (varying from $1 \%$ in young children to $6 \%$ in older children) (4). Among children, the incidence of COVID-19 disease was reported $12.3 \%$ (171 of 1391 children) in a study in China and $11.2 \%$ (41 of 365 children) in a study in Spain $(6,7)$. In our study, RT-PCR test was done in 835 children with suspected COVID-19 infection and the test was positive in $178(21.3 \%)$ children. The incidence of COVID19 disease in children in the community is 1-5\%, while the incidence of COVID-19 disease in children is approximately $10-20 \%$ in suspected pediatric cases.

Of the studies with reported that patients' mean age varied across studies from 6.2 to 11 years (8). In our study the mean age of patients who was confirmed diagnosis COVID-19 was 10.5 year. As can be seen, the incidence of COVID-19 is higher in older children. This situation can be explain that usually, younger children take part in fewer daily social activities than teenagers, and their contact opportunities with carriers or patients should thus be lower, resulting in a lower risk of getting the COVID-19 illness, as stated in a review (9).

In our study, a total of 28 COVID-19 patients were identified in approximately 3 months from the beginning to June 30, while 150 COVID-19 patients were identified in the next 2.5 months. This is may be due to the normalization and social life in our country starting from June. In addition, the increase in our cases during the summer season indicates that there is no seasonal difference in the pandemic outbreak.

It has been reported that, the presenting clinical symptoms of pediatric COVID-19 patients were fever and cough $(10,11)$. In our study, while fever was the most common symptom with $67 \%$, headache (41\%) was the more common symptom than cough $(37 \%)$. Other common symptoms were sore throat $(26 \%)$, fatigue $(23 \%)$, myalgia $(23 \%)$ and diarrhea $(21 \%)$. The reason for the excessive headache, fatigue and myalgia in 
our study may be due to the large number of older children who could express their symptoms. In our knowledge, our study is the first study conducted according to the number of symptoms, and it is seen that pediatric COVID-19 patients presents with an average of four symptoms.

In a systematic review of the literature, it was reported that there were no studies reported data about pediatric loss of smell or taste (8). In our study, $13 \%$ of the patients had loss of smell, and $9 \%$ of patients had loss of taste. There have been case reports of neurological complications in adults with COVID-19 including peripheral nervous disorders, but there has not been a clear indication that neurological complications predominate in children infected with COVID-19 $(4,12)$. In our study, a 17-year-old male patient had a left leg paralysis lasting for 12 days. In addition, five patients had atypical symptoms. These were hoarseness in one patient, backache in two patients, conjunctivitis in one patient, and painful swelling in the eyes in one patient. It is seen that especially adolescent children can present with very different symptoms.

The World Health Organization (WHO), Centers for Disease Control and Prevention (CDC) and several other government health agencies emphasize fever and respiratory symptoms in the criteria for suspected cases $(13,14,15)$, however, we detected in our study that children can have a wide range of symptoms. While $40 \%$ of the patients have gastrointestinal symptoms with fever or respiratory symptoms, $15 \%$ of the patients have only gastrointestinal symptoms without fever or respiratory symptoms.

In our study, Fifteen $(8.6 \%)$ of the patients were hospitalized, three $(1.7 \%)$ of them were required intensive care unit and one patient who had neurological comorbid disease was died from SARS-CoV-2 infection. Our study was similar to previous pediatric COVID-19 studies in terms of hospitalization, intensive care, and death $(16,17,18)$. Compared to adults, COVID-19 infection is seen to be milder and cause less hospitalizations and patient deaths in children $(11,16,19)$. The reason for this is remain elusive, some hypotheses have been proposed. First, children and adults have different immune systems. Children's weaker immune responses may prevent pulmonary destruction caused by virus-induced immune responses. Children are vulnerable to having many viral infections, and they can establish innate immune responses. In addition, various viruses simultaneously invading the airways and mucosa of the lungs may competitively inhibit SARS-CoV-2. Some researchers also suggested that children's innate immune responses are more active, and their respiratory tracts are healthier because of their low exposure to cigarette smoke and air pollution (10). Second, Ageing is associated with a progressive decline in the normal functioning of the immune system, which leads to weaker immune responses and impairs a person's ability to respond to new stimulants. This process involves the natural involution of the thymus, starting during or soon after the first year of birth. It then shows an accelerated decline after puberty, and the thymic microenvironment cells continue to reduce by $3 \%$ to $5 \%$ a year. After the fourth and fifth decade of life, the involution of the thymus leads to a significant decline in naïve $\mathrm{T}$ cell output. This affects the composition of the peripheral CD4 and CD8 T-cell pool and has a negative influence on the adaptive immunity that is considered to be the leading cause of morbidity and mortality in the elderly. It has been reported that, the number of $\mathrm{T}$ cells was significantly decreased in patients affected by COVID-19 disease, suggesting that dysregulation of the immune response involving $\mathrm{T}$ lymphocytes was present in this pathological infection (20). Third, SARS-CoV-2 enters respiratory epithelial cells by attaching to angiotensin converting enzyme-2 (ACE-2) via S-protein. After entering pneumocytes, SARS-CoV-2 downregulates ACE-2 expression, decreasing angiotensin-2 metabolism. Elevated angiotensin-2 increases pulmonary vascular permeability and inflammation, hence worsening of lung injury. Angiotensin-2 levels have been found to be increased in COVID-19 patients compared to healthy adults. ACE-2 levels decrease in old age and co-morbidities like hypertension and diabetes, possibly explaining worse lung injury and prognosis of SARS-CoV-2 in them. High levels of ACE-2 activity in children could be protective in children with COVID-19, leading to less severe disease in this age group compared to elderly (21).

Twenty (11.4\%) of our patients had asthma. Only two of the patients with asthma were hospitalized and didn't require intensive care unit. Our study supports an international study that states that children with asthma do not appear to be disproportionately affected by COVID-19 (22).

Sixty one percent of the patients were infected by close contact with family members diagnosed with COVID$19,20 \%$ of the patients were infected via close contact with non- family members diagnosed with COVID-19, 
$6 \%$ of the patients had a history of exposure in an epidemic area, including wedding hall, condolence house and shopping center and $13 \%$ of the patients with unknown source of infection, however, most of them had a history of using public transport. Therefore, we consider that especially crowded indoor environments are the main centers of the spread of the outbreak.

According to studies, it is reported that children are unlikely to be index cases and most of them acquire COVID-19 infection from adults and especially family members $(4,10,23,24,25)$. In our study, in half of the 68 patients whose source of infection was outside of family members, nobody had COVID-19 at home after them, but at least one person at home was infected with COVID-19 in the other half, however, most of them at the same time with the child had a history of close contact with non- family members diagnosed with COVID-19. In addition, at least one family member was hospitalized in 31 patients whose source of infection was family members and at least one family member was hospitalized in only one patients whose source of infection was outside of family members. Therefore, our study supports the systematic review concludes that children are unlikely to be the main drivers of the pandemic (26).

The mean age and mean symptoms of patients whose source of infection was outside of family members was more than whose source of infection was family members. It may be due to older children having more outside social activities and being able to express themselves better and be aware of their symptoms.

lymphocytopenia is reported to be associated with severe COVID-19 infection in adults (10). Guan et al studied 1099 adult patients and found the rate of lymphocytopenia was $83.2 \%$ (27). In a study conducted in Italy, lymphocytopenia was found in $15.7 \%$ of pediatric COVID-19 patients and increases in aspartate aminotransferase and alanine aminotransferase were reported in $18.3 \%$ and $11.8 \%$, respectively (28). In our study, lymphocytopenia was detected in $19.7 \%$ of the patients, however, liver enzymes were increased in very few patients.

In a study lung findings were detected in $27(46 \%)$ of the patients who underwent chest X- ray imaging. It was unilateral in 15 and bilateral in 12 patients. Localized increase in density was observed in 27 patients while a diffuse increase in density was observed in cases who were admitted to the intensive care unit (29). In our study, 52 patients were underwent chest X-ray. Lung findings were detected in 19 (36.5\%) patients. It was unilateral localized increase in density in seven patients on the right lower zone, bilateral localized increase in density in six, unilateral mild bronchial thickening in five patients on the right zone and bilateral diffuse increase in density in one patient.

\section{CONCLUSION}

We detected in our study that children with COVID-19 infection can have a wide range of symptoms. While $40 \%$ of the patients have gastrointestinal symptoms with fever or respiratory symptoms, $15 \%$ of the patients have only gastrointestinal symptoms without fever or respiratory symptoms. In our knowledge, our study is the first study conducted according to the number of symptoms, and it is seen that pediatric COVID-19 patients presents with an average of four symptoms. It is seen that especially adolescent children can present with very different symptoms. COVID-19 infection is seen to be milder and cause less hospitalizations and patient deaths in children. We consider that especially crowded indoor environments are the main centers of the spread of the outbreak and children are unlikely to be the main source of infection of the pandemic.

\section{CONFLICT OF INTEREST}

The authors have no financial relationships or other conflict of interests to disclose.

\section{REFERENCES}

1. Zare-Zardini H, Soltaninejad H, Ferdosian F et al. Coronavirus Disease 2019 (COVID-19) in Children: Prevalence, Diagnosis, Clinical Symptoms, and Treatment. Int J Gen Med. 2020; 13:477-482.

2. Tezer H, Demirbağ TB. Novel coronavirus disease (COVID-19) in children. Turk J Med Sci. 2020 ;50: 592-603. 
3. Sankar J, Dhochak N, Kabra SK et al. COVID-19 in Children: Clinical Approach and Management. Indian J Pediatr. 2020; 87: 433-442.

4. Williams PCM, Howard-Jones AR, Hsu P et al. SARS-CoV-2 in children: spectrum of disease, transmission and immunopathological underpinnings. Pathology. 2020; 3025: 30884-9.

5. Henry BM, Benoit SW, Santos de Oliveira MH et al. Laboratory abnormalities in children with mild and severe coronavirus disease 2019 (COVID-19): A pooled analysis and review. Clin Biochem. 2020; $81: 1-8$

6. Lu X, Zhang L, Du H, et al. SARS-CoV-2 infection in children. N Engl J Med. 2020; 382:1663-1665.

7. Tagarro A, Epalza C, Santos M et al. Screening and severity of coronavirus disease 2019 (COVID-19) in children in Madrid, Spain. JAMA Pediatr 2020;1346

8. Patel NA. Pediatric COVID-19: Systematic review of the literature. Am J Otolaryngol. 2020. 41(5):102573.

9. Jeng MJ. Coronavirus disease 2019 in children: Current status. J Chin Med Assoc. 2020; 83(6):527533.

10. Ding Y, Yan H, Guo W. Clinical Characteristics of Children With COVID-19: A Meta-Analysis. Front Pediatr. 2020; 8: 431.

11. Guo CX, He L, Yin JY et al. Epidemiological and clinical features of pediatric COVID-19. BMC Med. 2020. 18(1):250.

12. Helms J, Kremer S, Merdji H,et al. Neurologic features in severe SARS-CoV-2 infection.N Engl J Med2020; 382: 2268-70.

13. de Souza TH, Nadal JA, Nogueira RJN et al. Clinical manifestations of children with COVID-19: A systematic review. Pediatr Pulmonol. 2020. 55(8):1892-1899.

14. CDC Health Alert Network. Updated guidance on evaluating and testing persons for coronavirus disease 2019 (COVID-19). 2020.

15. World Health Organization. Global Surveillance for human infection with coronavirus disease (COVID19). 2020.

16. Ludvigsson JF. Systematic review of COVID-19 in children shows milder cases and a better prognosis than adults. Acta Paediatr. 2020. 109(6):1088-1095.

17. Dong Y, Mo XI, Hu Y, et al. Epidemiological characteristics of 2143 pediatric patients with 2019 coronavirus disease in China. Pediatrics. 2020;16:16

18. Bialek S, Boundy E, Bowen V, et al. Severe outcomes among patients with coronavirus disease 2019 (COVID-19) - United States, February 12-March 16, 2020. MMWR Morb Mortal Wkly Rep. 2020;69:343-346.

19. Zhang Y. The Epidemiological Characteristics of an Outbreak of 2019 Novel Coronavirus Diseases (COVID-19) - China, 2020. Chinese Journal of Epidemiology (by The Novel Coronavirus Pneumonia Emergency Response Epidemiology Team). 2020.

20. Ruggiero A, Attinà G, Chiaretti A. Additional hypotheses about why COVID-19 is milder in children than adults. Acta Paediatr. 2020; 109(8):1690.

21. Dhochak N, Singhal T, Kabra SK et al. Pathophysiology of COVID-19: Why Children Fare Better than Adults? Indian J Pediatr. 2020; 87(7):537-546.

22. Papadopoulos NG, Custovic A, Deschildre A et al. Impact of COVID-19 on Pediatric Asthma: Practice Adjustments and Disease Burden. J Allergy Clin Immunol Pract. 2020. 8(8):2592-2599.

23. Lee B, jr Raszka WV. COVID-19 Transmission and Children: The Child Is Not to Blame. Pediatrics. 2020. 146(2):e2020004879.

24. Götzinger F, Santiago-García B, Noguera-Julián A et al. COVID-19 in children and adolescents in Europe: a multinational, multicentre cohort study. Lancet Child Adolesc Health. 2020. 4(9):653-661.

25. Ciuca IM. COVID-19 in Children: An Ample Review. Risk Manag Healthc Policy. 2020; 13:661-669.

26. Jonas F Ludvigsson. Children are unlikely to be the main drivers of the COVID-19 pandemic - A systematic review. Acta Paediatr. 2020. 109(8):1525-1530.

27. Guan WJ, Ni ZY, Hu YU, et al. Clinical characteristics of coronavirus disease 2019 in China. N Engl J Med. 2019;2020 
28. Parri N, Magistà AM, Marchetti F et al. Characteristic of COVID-19 infection in pediatric patients: early findings from two Italian Pediatric Research Networks. Eur J Pediatr. 2020. 179(8):1315-1323.

29. Palabiyik F, Kokurcan SO, Hatipoglu N et al. Imaging of COVID-19 pneumonia in children. Br J Radiol. 2020. 93(1113):20200647.

\section{Hosted file}

Table.pdf available at https://authorea.com/users/365213/articles/485394-symptoms-andtransmission-of-covid-19-infection-in-children 